\title{
Brot und Spiele
}

\section{Die Rollen des Essens im psychodramatischen Fokus}

\author{
Sabine Spitzer-Prochazka
}

Online publiziert: 23. Oktober 2018

(C) Springer Fachmedien Wiesbaden GmbH, ein Teil von Springer Nature 2018

Liebe Leserin, lieber Leser,

ich möchte Sie gerne einladen, folgendes Bild auf Ihrer inneren Bühne entstehen zu lassen:

Ein reich gedeckter Tisch im Schatten eines ausladenden Apfelbaums, üppig dekoriert mit bunten Blumen, deren Farben sich auf den kunstvoll gefalteten Servietten wiederfinden. Im geflochtenen Weidenkorb verstecken sich knusprige Gebäckstücke, reichlich bestreut mit Sesam, Mohn oder Rosmarinsalz, in der Schüssel daneben stapeln sich goldgelbe Birnen, saftige Melonenstücke, überreife Pfirsiche und leuchtendrote Himbeeren. Nebenan eine Platte mit kaltem Braten (sehen Sie die krosse, pikant gewürzte Kruste?), rosafarbene Schinkenrollen, fein durchzogene Wurstscheiben neben großlöchrigem Emmentaler und cremigem Weichkäse. Farbenfroh harmoniert das Rote-Rüben-Carpaccio mit dem steirischen Käferbohnen-Salat. Der Altwiener Guglhupf trägt eine dicke Schicht aus Staubzucker, daneben warten hübsche Croissants auf die selbstgemachten Marmeladen. Dazu passt der erfrischende, eiskalt aufgeschäumte Lavendelsirup. Falls Sie Tee bevorzugen: die bauchigen Kannen halten anregende Aromen für Sie bereit ....

Wenn Sie mir bis hierher gefolgt sind, woran denken Sie gerade? Vielleicht hat der süße Kuchen Erinnerungen geweckt? Gut möglich, dass Sie Ihre längst verstorbene Großmutter sehen, wie sie in der Blumenschürze vor dem Backrohr steht und den Handmixer schwingt. Oder gab es bei Ihnen immer sonntags Braten, und die ganze Familie versammelte sich um den Mittagstisch? Gut möglich, dass Onkel Gebhard

S. Spitzer-Prochazka $(\square)$

Schwaigergasse 35/25, 1210 Wien, Österreich

E-Mail: therapeutin@aon.at 
und Tante Mitzi noch vor der Suppe so innig zu streiten begonnen haben, dass bis zum Hauptgang sämtliche Familienmitglieder in die Kontroversen involviert waren - aber spätestens beim Dessert alle wieder gut miteinander waren.

Oder haben Sie Hunger verspürt, angesichts unseres reich gedeckten Tisches? Wann haben Sie eigentlich zuletzt gegessen, was und wie viel? Haben Sie achtsam Bissen für Bissen genossen oder in atemberaubender Geschwindigkeit alles hinuntergeschlungen? Gut möglich, dass Sie beim Anblick der Speisen blitzschnell, weil routiniert, das Mischungsverhältnis aus Kohlehydraten und Eiweiß überprüft, den Fettgehalt gecheckt und die Kalorien addiert haben. Um gleich danach die nächste Sporteinheit zu planen (um das Croissant zu verbrennen, müssten Sie 28 min joggen oder 38 min Yoga machen ...). Gut möglich aber auch, dass Sie unserem Buffet angeekelt den Rücken kehren, weil es alles andere als vegan gestaltet ist.

Das Essen in all seinen Facetten berührt uns auf sämtlichen Rollenebenen: es beeinflusst unsere körperliche Verfassung, weckt und dämpft Gefühle, ist wichtiges Bindemittel sozialer Beziehungen und wird oftmals zur Glaubensfrage. Es begleitet uns ein Leben lang: vom Säuglingsbrei der ersten bis zum Brei der späten Jahre im Altersheim spiegelt unsere Nahrung auch Abhängigkeiten wieder. So mancher Kampf um Autonomie spielt sich am Familienesstisch ab und wird über die Nahrung ausgefochten. In allen Schattierungen kann das Essverhalten problematisch werden oder gestört sein, ein Zuviel oder Zuwenig, Einseitigkeit oder generelle Verweigerung bieten ausreichend Konfliktstoff für die Beziehung zum eigenen Körper.

Glücklich schätzen kann sich, wer mit obigem Buffet einfach einen schönen Sonntagmorgen beim Brunch mit den Liebsten verbindet, und genießen kann, den Moment, die Begegnung und die wunderbaren Nahrungsmittel. Nicht umsonst gilt das (Wieder)Entdecken der Genussfähigkeit als ein wesentliches Ziel in der Psychotherapie. Davon und von anderen Themen rund ums Essen handeln die Beiträge in diesem Sonderband, für den Autorinnen und Autoren aus Deutschland, Österreich und der Schweiz ihren psychodramatischen Erfahrungsschatz zur Verfügung gestellt haben.

Den Sonderband eröffnet Julia Ortner mit einer soziodramatischen Betrachtung der Ernährung. Als gebürtige Kärntnerin lebt die Autorin seit mehr als zwei Jahrzehnten in Wien, sie war unter anderem in der Sozial- und Jugendarbeit sowie in der Film- und Werbebranche tätig, bevor sie ihre Psychotherapiepraxis in Wien eröffnete. In ihrem Artikel geht sie bis in die Steinzeit zurück, um die Entwicklung der aktuellen Ernährungstrends nachzuzeichnen - Trends, deren es immer mehr zu geben scheint und die sie in ihrer Auflistung erklärt: Von Veganismus und Clean Eating, über Super- und Raw Food, bis hin zu Low- und gänzlich Free-Carb reichen die Ernährungsgewohnheiten der heutigen Zeit. In einer „Gesellschaft der OptimiererInnen“, so Ortner, ,,wird die intensive Beschäftigung mit der Ernährung zur Pflicht“.

Lisa Tomaschek-Habrina ist - unter anderem - Mitbegründerin eines Instituts für Burnout- und Stressmanangement in Wien und Betreiberin eines Blogs zu allen Themen rund um Burnout und dessen Behandlungsmöglichkeiten. In ihrem Beitrag stellt sie Zusammenhänge zwischen Erschöpfungsprozessen und der Ernährung her und erläutert, wie sie betroffene KlientInnen mittels mehrdimensionalen Behandlungs- 
konzepts begleitet und dabei unterstützt, jene Lebensgewohnheiten zu verändern, die zur Erschöpfung geführt haben.

Die Unternehmensberater Christoph Buckel und Uwe Reineck, beide vom Psychodrama-Institut Freiburg/Heidelberg, gestalten Lernreisen, das sind mobile Seminare mit dem Ziel, die TeilnehmerInnen szenisch mit unbekannteren Welten zu konfrontieren, um ihnen neue Perspektiven auf die eigene Rollengestaltung zu ermöglichen. Im Rahmen dieser ganzheitlichen, reich- und nachhaltigen Form von Lernen und Persönlichkeitsentwicklung spielen Szenen des Essens eine besonders nahrhafte Rolle, weil: „Sie aktivieren basale Rollen, die sonst häufig ausgeblendet werden. Sie schaffen Atmosphäre, in der es sich leichter vertrauen und leichter verdauen lässt.“, so die Autoren.

Ein psychodramatisches Arrangement, das sie vollständig aus Szenen des Essens entwickelt hat, präsentiert Daniela Trattnigg in ihrem Beitrag über den sinnlichen Koch_Dialog. Die Kärntner Psychodramatikerin und zusätzlich ausgebildete Sexualtherapeutin bezeichnet sich selbst als Lebenslustköchin und zieht zahlreiche Parallelen zwischen Kochen, Essen und Sexualität. Letztere müsse ,ebenso wie Kochen gelernt werden, damit sie schließlich zur Kunstform wird und auf mehreren Ebenen erfüllend gelebt werden kann“, postuliert sie in ihrem Beitrag.

Das Lebkuchenhaus, den süßen Brei, Schneewittchens vergifteten Apfel - wer kennt sie nicht, die anschaulichen, symbolstarken Nahrungsmittel aus den Überlieferungen der Gebrüder Grimm. Märchenhafte Essens-Szenen serviert uns Regina Bulian, Psychotherapeutin und Pädagogin aus der Steiermark, wenn sie in ihrem Artikel über die Inszenierung verschiedener Märchenstoffe schreibt. In einem Beispiel lässt sie die TeilnehmerInnen einer Jahresgruppe ein neues Gruppenmärchen entwickeln, indem jedeR die eigene Lieblingsfigur auf die Bühne bringt. Im Feedback und Sharing zu diesem Spiel wird erkennbar, welche Themen die DarstellerInnen der Prinzessin auf der Erbse, vom Wolf sowie der Hexe aus Hänsel und Gretel im Alltag gerade beschäftigen.

Spielerisch geht es auch im nächsten Beitrag weiter: Christian Stadler und Sabine Spitzer-Prochazka, die beiden HerausgeberInnen der Zeitschrift für Psychodrama und Soziometrie, haben verschiedene Arrangements gesammelt, die sich der Symbolik des Essens bedienen und in Beratung und Therapie eingesetzt werden können, um Gruppenprozesse in Gang zu bringen.

Findet der Alltag vorwiegend im Krankenhaus statt und ist er geprägt von lebensbedrohlichem Kranksein, kommt der Ernährung wiederum eine besondere Rolle zu. Über ihren Zugang in der Rolle der Psychoonkologin schreibt Birgt Zilch-Purucker: „Wenn die Begegnung tragend ist, dann kann über die Themen Ernährung und Essen sich das Existentielle öffnen: Gespräche über Tod und Abschied, Autonomie und Abhängigkeit, Zwänge und Freiheit in den zwischenmenschlichen Beziehungen, nur um die wichtigsten zu benennen." Die Autorin ist ärztliche Psychotherapeutin und hat langjährige Erfahrung in der Beratung und Betreuung krebskranker Menschen und deren Angehöriger. Sie arbeitet im evangelischen Krankenhaus in Wesel.

Der zweite Teil des Sonderbandes widmet sich dem Thema Essstörungen. Damit diese gar nicht erst entstehen, setzt die Präventionsarbeit schon in Kindergarten und Schule an. „Die Förderung von Lebenskompetenz wird international als wirkungsvollste (sucht-)präventive Maßnahme bei Kindern und Jugendlichen ein- 
gesetzt“, schreibt Christine Pichlhöfer in der Einleitung ihres Beitrags „Wann bin ich richtig?" und erläutert den Aufbau und Inhalt jener Präventions-Workshops, die sie an verschiedenen Schulen leitet. Als Ursachen für Essstörungen ortet sie neben anderen Faktoren auch gesellschaftliche Entwicklungen wie den Trend zur „Körperoptimierung“. Die Psychotherapeutin und Sozialarbeiterin ist seit den 90er Jahren in der Suchtarbeit tätig, unter anderem ist sie Projektleiterin in der Suchtprävention in Niederösterreich und Wien.

Besonders erfahren und spezialisiert in der Suchtarbeit ist auch Bettina WaldhelmAuer, die in eigener Praxis in Salzburg tätig ist und zahlreiche Lehraufträge für Suchttherapie innehat. Ausgehend vom Fallbeispiel einer Bulimie-Patientin zeigt die Autorin die Entwicklungsschritte auf dem Weg zu einem gesunden Essverhalten auf: „Zum Wiedererlernen normalen Essverhaltens gehören die Integration des Körpers, die Wahrnehmung abgespaltener Gefühle und perfekter Ziele, sowie die Förderung entsprechender Rollen der körperlichen Fürsorglichkeit.“, zählt sie in ihrem Beitrag auf.

Warum sich Musiktherapie so gut für den Einsatz in der Behandlung von Essstörungen eignet und wie diese im Rahmen einer Psychodramatherapie eingesetzt werden kann, beschreibt Heidi Fausch-Pfister in ihrem Artikel. Neben der Arbeit in ihrem Atelier für Musiktherapie und Psychodrama im Schweizer Kanton Aargau lehrt und publiziert sie international. „Musik berührt ohne Körperkontakt“, schreibt sie in ihrem Beitrag und resümiert: „Psychodrama und Musiktherapie sind ein starkes Paar.“

Menschen mit Essstörungen leiden oft zusätzlich an weiteren Erkrankungen, wie Depressionen, posttraumatischen Belastungs- oder Persönlichkeitsstörungen - diesem Phänomen der Komorbidität widmet sich Susanne Kunz-Mehlstaub in ihrem Beitrag. Die Psychotherapeutin und Fachärztin für Psychiatrie, die ihre Ausbildung in Österreich und der Schweiz absolviert hat, lebt in St. Gallen und hat kürzlich, gemeinsam mit Christian Stadler, ihr erstes Buch veröffentlicht.

Abschließend führt uns Ulrike Altendorfer-Kling an die Schnittstelle zwischen Medizin und Psychotherapie. Anhand eines Fallbeispiels zeigt sie auf, welche komplexen Herausforderungen die stationäre Behandlung einer Jugendlichen mit Anorexie an die BehandlerInnen beider Disziplinen stellt und wo die Gefahren einer Rollenkonfusion liegen. Die Autorin ist Fachärztin am Salzburger Landesklinikum, Leiterin der Kinderseelenhilfe Salzburg und hat verschiedene Lehraufträge inne.

Trotz der Fülle an Beiträgen liegt eine unfreiwillig abgespeckte Version dieses Sonderbandes vor Ihnen, weil zahlreiche KollegInnen ihre zugesagten Beiträge leider nicht abgeliefert haben. Etliche Zusagen wurden im allerletzten Moment noch zurückgezogen, sodass es auch nicht mehr möglich war, rechtzeitig Ersatz zu finden. Damit sind viele weitere spannende Aspekte des Essens-Themas leider buchstäblich unter den Tisch gefallen. Umso mehr: ganz herzlichen Dank an jene Autorinnen und Autoren, die sich durchgebissen und uns die Lektüre schmackhaft gemacht haben!

Übrigens: falls Sie tatsächlich Lust auf die Speisen unseres eingangs imaginierten Buffets bekommen haben - Sie finden einige Rezepte zum Nachkochen in diesem Heft. Wir haben die Autorinnen und Autoren gebeten, ihre Lieblingsrezepte zu verraten und haben diese im Anschluss nach jedem Artikel abgedruckt. In diesem Sinne wünsche ich Ihnen diesmal nicht nur viel Freude beim Lesen, sondern auch 
beim Nachkochen und Genießen von Rote-Rüben-Carpaccio, Schweinebraten und mehr.

\section{Guten Appetit!}

S. Spitzer-Prochazka

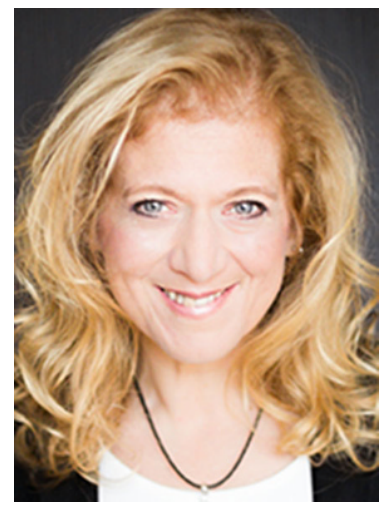

Sabine Spitzer-Prochazka, Jg. 1968, Psychodrama-Psychotherapeutin in freier Praxis in Wien, Gruppentherapeutin in der psychotherapeutischen Ambulanz des ÖAGG, Redakteurin und Mitherausgeberin der Zeitschrift für Psychodrama und Soziometrie. 